\title{
Magnetic field structures inside magnetars with strong toroidal field
}

\author{
Kotaro Fujisawa \\ Department of Earth Science and Astronomy, Graduate School of Arts and Sciences, \\ University of Tokyo, Komaba, Meguro-ku, Tokyo 153-8902, Japan \\ email: fujisawa@ea.c.u-tokyo.ac.jp
}

\begin{abstract}
We have analyzed the magnetized equilibrium studies with strong toroidal magnetic fields and found that the negative toroidal current density inside the star is very important for the strong toroidal magnetic fields. The strong toroidal magnetic fields require the strong poloidal current, but the strong poloidal current results in the localized strong toroidal current density in the axisymmetric system. This localized toroidal current changes the magnetic field configuration and makes the size of the toroidal magnetic field region smaller. As a result, the toroidal magnetic field energy can not become large. We need to cancel out the localized toroidal current density in order to obtain the large toroidal fields solutions. We have found and showed that the negative toroidal current cancels out the localized toroidal current density and sustain the large toroidal magnetic field energy inside the star. We can explain the magnetized equilibrium studies with strong toroidal magnetic fields systematically using the negative current density. Physical meaning of the negative current is key to the magnetar interior magnetic fields.
\end{abstract}

Keywords. Stars: interior - Stars: neutron - Stars: magnetic fields

\section{Introduction}

Anomalous X-ray Pulsars (AXPs) and Soft Gamma-ray Repeaters (SGRs) are considered as special classes of magnetized neutron stars, magnetars. Magnetars have very strong dipole magnetic fields whose typical values reach about $10^{15} \mathrm{G}$ at their surfaces. Recent observations, however, reported the low magnetic fields SGRs whose dipole fields are much lower than typical values (Rea et al. 2010). Their high energy activities would be driven by the internal strong toroidal magnetic fields energy, but we can not observe the internal magnetic fields directly. We need to calculate magnetized stars in equilibrium theoretically to obtain the internal magnetic fields configurations.

Many theoretical studies, however, failed to obtain the magnetized star with strong toroidal magnetic fields (e.g. Ciolfi et al. 2009; Lander \& Jones 2009; Fujisawa et al. 2012 etc.). The typical value of toroidal to total magnetic filed energy ratio $M_{t} / M$ is only a few percent. On the other hand, only a few works had obtained the strong toroidal models under special boundary conditions. Glampedakis et al. (2012) obtained strong toroidal field models $\left(M_{t} / M \sim 25 \%\right)$ imposing surface current on the stellar surface. Duez \& Mathis (2010) obtained very strong toroidal models $\left(M_{t} / M \sim 66 \%\right)$ imposing the boundary condition that the magnetic flux on the stellar surfaces should vanish. Therefore, all magnetic field lines in the model are closed and confirmed within the star. These works, however, did not describe the reason why such boundary conditions result in the strong toroidal magnetic fields. In order to answer this question, we have analysed these works carefully and found the negative toroidal current density (opposite flowing toroidal current density) inside the star plays very important role for the strong toroidal magnetic fields (see Fujisawa \& Eriguchi 2013). 


\section{Formulation and arbitrary functions}

We assume that the system is stationary and axisymmetry. Then, the magnetic fields are described by two scalar functions $\Psi$ and $I$ as below:

$$
\boldsymbol{B}=\frac{1}{r \sin \theta} \nabla \Psi(r, \theta) \times \boldsymbol{e}_{\varphi}+\frac{I(r, \theta)}{r \sin \theta} \boldsymbol{e}_{\varphi}
$$

where $\Psi$ is a poloidal magnetic flux function and $I$ is a poloidal current flux function. The magnetic flux function $\Psi$ is governed by the equation as

$$
\frac{\partial^{2} \Psi}{\partial r^{2}}+\frac{\sin \theta}{r^{2}} \frac{\partial}{\partial \theta}\left(\frac{1}{\sin \theta} \frac{\partial \Psi}{\partial \theta}\right)=-4 \pi r \sin \theta \frac{j_{\varphi}}{c}
$$

The source term of this equation is the toroidal current density in the right hand side. The toroidal current density is determined by matter equation. From the axisymmetric condition, $I$ is a conserved quantity along the magnetic field line $(I \equiv I(\Psi))$ in both barotropic and baroclinic systems (see Braithwaite 2009). If the magnetized star is barotropic $(p=p(\rho))$ without differential rotation and meridional flow, the toroidal current density is described using two arbitrary function $I$ and $F$ as

$$
\frac{j_{\varphi}}{c}=\frac{1}{4 \pi} \frac{d I(\Psi)}{d \Psi} \frac{I(\Psi)}{r \sin \theta}+\rho r \sin \theta F(\Psi) .
$$

Where $F$ is also a conserved quantity $(F \equiv F(\Psi))$ which is related to the Lorentz force (see Fujisawa et al. 2012). If the outside of the star is vacuum, $I$ must vanish in the region. As follow many previous studies, we fix the functional forms of $F$ and $I$ as

$$
F(\Psi)=F_{0}, \quad I(\Psi)=\frac{I_{0}}{k+1}\left(\Psi-\Psi_{\max }\right)^{k+1} \Theta\left(\Psi-\Psi_{\max }\right),
$$

where $F_{0}$ and $k$ are constants and $\Psi_{\max }$ is a maximum value of $\Psi$ in the vacuum region. We fix $k=0.1$. $I_{0}$ means the local strength of the toroidal magnetic field. $\Theta$ is Heaviside-step function. We calculate Eq. (2.2) using Green function relaxation method with arbitrary current sheets. (see Fujisawa \& Eriguchi 2013).

\section{Results \& Discussion}

First, we have calculated three magnetized equilibrium states changing the value of $I_{0}$. The numerical solutions are displayed in Fig 1. The top panels show the poloidal magnetic field configurations and the strength of the toroidal magnetic fields. The bottom panels show the distributions of the toroidal current density. As the value of $I_{0}$ increases, the local strength of the toroidal magnetic field also increases. At the same time, however, the size of the toroidal magnetic field region becomes smaller.

The strong toroidal magnetic fields require the strong poloidal current $I$ (Eq. 2.1), but $I$ is related to the toroidal current density by the first term in Eq. (2.3). As the $I$ increases, the toroidal current density also increases. Since the size of the toroidal magnetic field region is limited by the boundary condition, the toroidal current from the $I$ term is localized within the stellar interior (see Fig. 1). The localized strong toroidal current changes the magnetic fields configurations. The distribution of $\Psi$ also becomes localized and the size of the toroidal field region becomes smaller (Fig. 1). As a result, we fail to obtain the large toroidal magnetic fields solutions. We see similar results as Fig. 1 in the previous studies (see table 2 in Lander \& Jones 2009, Fig. 12 in Ciolfi et al. 2009, Fig. 4 in Glampedakis et al. 2012). Therefore, this behaviour of the toroidal field region is a general feature of axisymmetric magnetized equilibria. We need to cancel out the localized toroidal current from the $I$ term to obtain the strong toroidal magnetic models. 

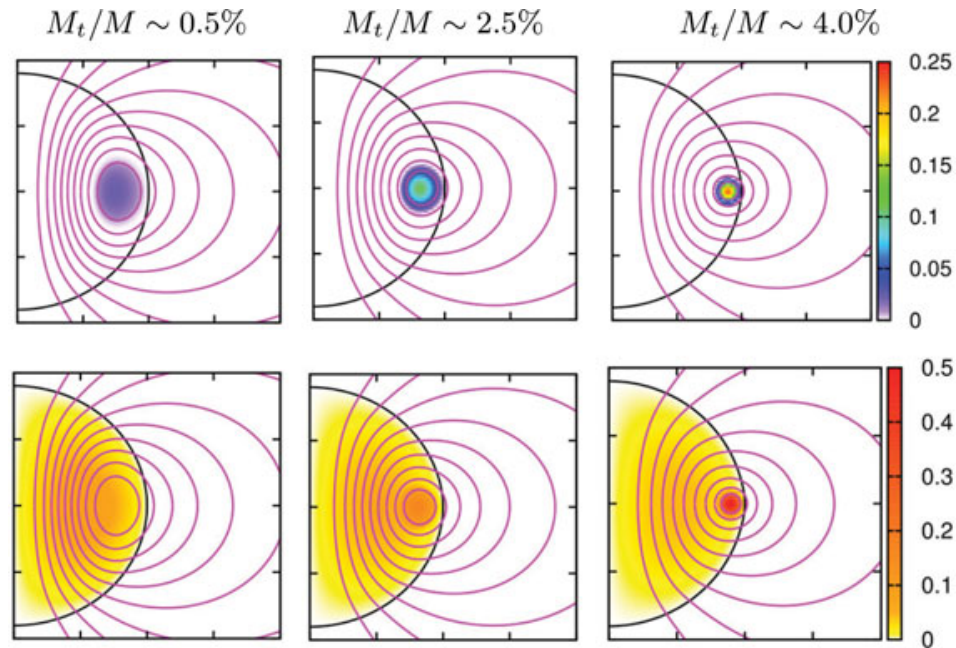

Figure 1. The magnetic field configurations and the strengths of the toroidal magnetic fields (colour maps, top) and the distributions of toroidal current density (color maps, bottom). Each panel shows $I_{0}=5$ (left), $I_{0}=20$ (center) and $I_{0}=40$ (right) solution respectively.
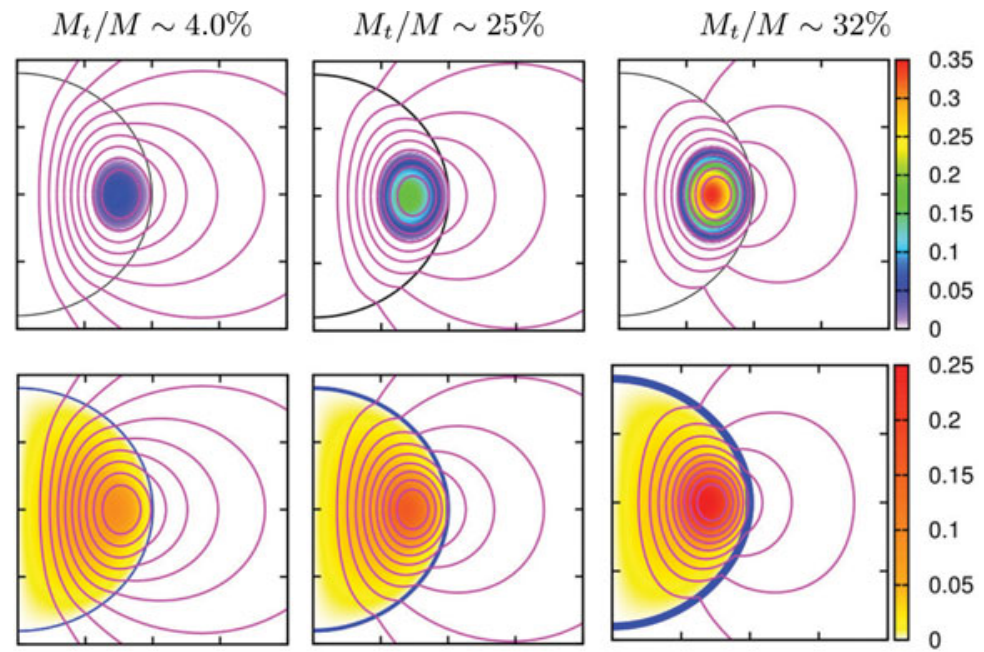

Figure 2. Same as Fig. 1 except for the negative surface current. The width of the surface represents the surface current strength. Each panel shows the solution with weak surface current (left), middle surface current (center) and strong surface current (right).

The surface current can cancel out the toroidal current density from the $I$ term. Next, we have calculated the magnetized equilibrium states with negative surface current (Fig. 2). The negative surface current cancel out the localized toroidal current density and the size of the toroidal magnetic fields does not become small (see from left to right panel). As a result, these models can sustain the strong toroidal magnetic fields energy and the value of the energy ratio reaches $M_{t} / M \sim 32 \%$.

We can explain the magnetized equilibrium states with strong toroidal magnetic fields systematically using the negative toroidal current. We classify the previous studies into three types according to the boundary conditions at the surface, (a): open fields model (e.g. Tomimura \& Eriguchi 2005; Lander \& Jones 2009; Ciolfi et al. 2009), (b): surface current model (Glampedakis et al. 2012) and (c): close fields model (Duez \& Mathis 


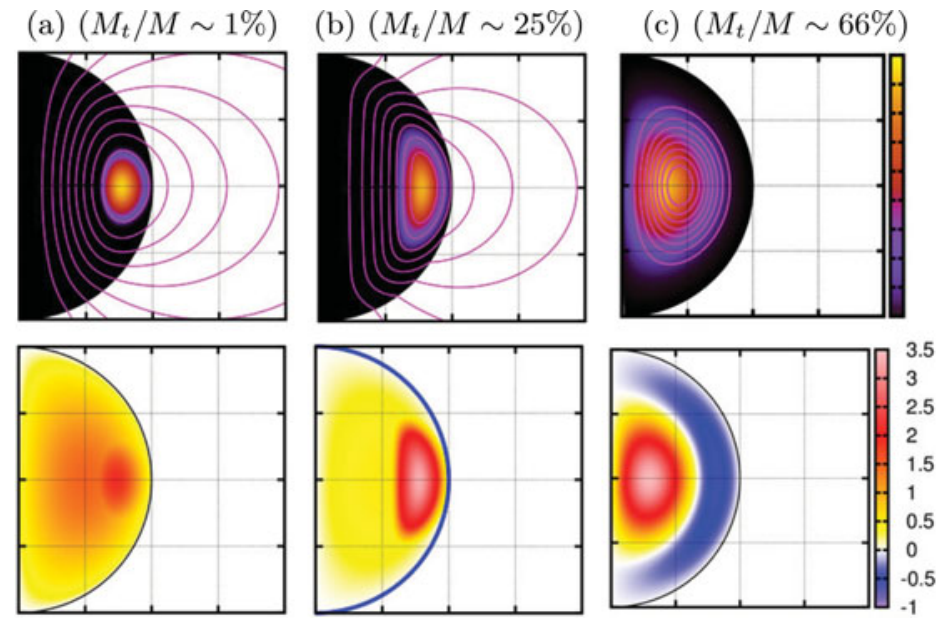

Figure 3. Top: The poloidal magnetic field lines and the toroidal magnetic field strength (color map). Bottom: The toroidal current density distributions (color map) of each model.

2010; Yoshida et al. 2012). The panels in Fig. 3 display the magnetic fields configuration (top) and the toroidal current density (bottom) in each model. The typical energy ratios are $M_{t} / M \sim 1 \%$ (a), $\sim 25 \%$ (b) and $\sim 66 \%$ (c). (b) and (c) have the strong toroidal magnetic fields energy because they have the negative toroidal current density. As seen from Fig. 2, (b) has the negative surface current and (c) has the negative toroidal current density region near the stellar surface, while (a) does not have the negative toroidal current inside the star. These results show the negative toroidal current are required to sustain the strong toroidal magnetic fields inside the star.

Very recently, Ciolfi \& Rezzolla (2013) have succeed in obtaining the magnetized equilibria with very strong toroidal magnetic energy $\left(M_{t} / M \sim 90 \%\right)$. We can also explain their solutions using the negative current density. They changed the functional form of $F$ in order to cancel out toroidal current more effectively (see the bottom panels of Fig. 2 in the paper). The negative toroidal current density plays also important role in this case.

It is important to consider the physical meanings of the negative current. One possibility is magnetic stress on the bottom of the crust (Braithwaite \& Spruit 2006). Our results show that the strong core toroidal fields are sustained by the crust of the magnetar. Physical meaning of the negative current is key to the magnetar interior fields.

\section{References}

Braithwaite, J., 2009, MNRAS, 397, 763

Braithwaite, J. \& Spruit, H. C., 2006, A\&A, 450, 1097

Ciolfi, R., Ferrari, V., Gualtieri, L., \& Pons, J. A., 2009, MNRAS, 397, 913

Ciolfi, R. \& Rezzolla, L., 2013, MNRAS, 435, L43

Duez, V. \& Mathis, S., 2010, A\&A, 517, A58

Fujisawa, K. \& Eriguchi, Y., 2013, MNRAS, 432, 1245

Fujisawa, K., Yoshida, S., \& Eriguchi, Y., 2012, MNRAS, 422, 434

Glampedakis K., Andersson N., Lander S. K., 2012,MNRAS, 420, 1263

Lander, S. K. \& Jones, D. I., 2009, MNRAS, 395, 2162

Rea, N. et al., 2010, Science, 330, 944

Tomimura, Y. \& Eriguchi, Y., 2005, MNRAS, 359, 1117

Yoshida, S., Kiuchi, K., \& Shibata, M., 2012, Phys. Rev. D, 86, 044012 